\title{
Translational control decrypted
}

\section{Recent research efforts have made great strides in elucidating the process, machinery and mechanisms that control how mRNAs are decoded by ribosomes in the process of protein translation.}

$\mathbf{T}$ The Enigma machine (a detail of which was used in this issue's cover image) was invented early in the twentieth century and used to encrypt and decrypt secret messages, most notably by the German military before and during Word War II. British code breakers famously managed to decrypt a large number of Enigma-enciphered messages, and the intelligence gleaned from this information is thought to have aided the Allied war effort. In a manner somewhat conceptually similar to the Enigma machine, ribosomes 'decrypt' - or decode-by scanning the bases along mRNAs and selecting aminoacyl-tRNAs according to the codon sequence, resulting in the addition of amino acids to a nascent polypeptide chain. The recent structural elucidation of whole $80 \mathrm{~S}$ eukaryotic ribosomes by X-ray crystallography has provided a vast body of information on the translation machinery and the various functional sites in the small and large subunits. Comparing these structures with previously determined structures of bacterial ribosomes has uncovered bacteria- and eukaryote-specific features, the functional implications of which we can now start to analyze. Importantly, the structure determination of eukaryotic ribosomes will facilitate future studies of translation initiation and its regulation, of ribosome biogenesis and of ribosome-associated mRNA decay and protein degradation.

In this issue, we present a special Focus on Translational Control (http://www.nature.com/nsmb/focus/translation/index.html). The Focus kicks off with a Review by Yusupov and colleagues describing bacterial and eukaryotic ribosome structures, highlighting selected differences and their functional implications (page 560).

Translation initiation is the most highly regulated phase of the translation cycle. In eukaryotes, it involves the formation of the preinitiation complex (PIC), which includes the 40 S ribosomal subunit and associated initiation factors, in an 'open' conformation and its recruitment to the mRNA. Once loaded on the mRNA, the open PIC scans the mRNA to locate the start codon. Its recognition triggers a series of events prompting a transition to the closed PIC conformation, which arrests the scanning complex and ultimately leads to formation of the $80 \mathrm{~S}$ initiation complex. The complexity of the translation initiation pathway provides many opportunities for regulation, so understanding the molecular mechanics of individual steps is vital. In their Review, Echeverría Aitken and Lorsch (page 568) outline recent insights into key molecular events in this critical phase of gene expression and discuss important outstanding challenges.

The poly(A) tail at the $3^{\prime}$ end of the mRNA forms a closed loop with the $5^{\prime}$ cap structure, to which the PIC is recruited during translation initiation. The integrity of this circularized mRNA loop is a target for general translational control. In addition, transcript-specific translational control is determined by cis-acting regulatory elements, located mostly in the $3^{\prime}$ untranslated region (3' UTR) of mRNAs.
These regulatory elements assemble a myriad of regulatory factors that modulate the length of the poly(A) tail, which in turn defines the extent of mRNA translation. Méndez and colleagues (page 577) provide fascinating insight into the mechanisms that control poly(A) tail length and their coordination to modulate gene expression in physiologically important processes such as inflammation, learning and memory acquisition, and early development.

One group of regulatory elements in the $3^{\prime}$ UTR of mRNAs are microRNA (miRNA) target sites. miRNAs mediate gene silencing through the miRNA-induced silencing complex (miRISC) machinery, which interacts with various trans-acting factors that control gene expression by inhibiting translation and/or initiating mRNA decay. How exactly miRISC elicits those events, the order in which they take place and the proteins recruited by miRISC remain controversial topics. In their Review on page 586, Fabian and Sonenberg guide us through the latest discoveries and insights into these issues. Notably, two recent papers (Science 336, 233-237 (2012); Science 336, 237-240 (2012)) analyze the kinetics of miRNA-mediated gene silencing in fly cells and zebrafish embryos and demonstrate that miRNAs may act through a two-step mode of repression-first inhibiting translation in a deadenylation-independent manner, and then subsequently causing deadenylation, decapping and destabilization of targeted mRNAs. It remains to be determined whether deadenylation can proceed before translational repression, or whether the two events are mechanistically coupled.

The intricate link between translational repression and mRNA decay in miRNA-mediated gene silencing reflects a general theme in gene regulation. However, discussions of mRNA surveillance mechanismswhich monitor mRNAs as they are translated and result in the degradation of troublesome transcripts-have traditionally focused only on the effects on mRNAs. But given that these surveillance events take place on the ribosome, thereby directly implicating translation in these processes, it is important to consider the consequences for the translational output of a cell. In a thought-provoking Perspective on page 594, Shoemaker and Green explore mRNA surveillance from the viewpoint of its origins on the ribosome, which should lead to new insights that will inform future research.

Much like the code breakers of the early twentieth century, with recent breakthroughs researchers can now begin deciphering how the translational machinery functions and is regulated. We have collected some of the most timely papers in the field of translational control published by Nature Publishing Group in a library (http://www.nature. $\mathrm{com} / \mathrm{nsmb} /$ focus/translation/library/index.html) for your convenience. Last but not least, we would like to thank the contributors to this Focus for their excellent work in decrypting a very complicated but essential biological system. Happy reading! 